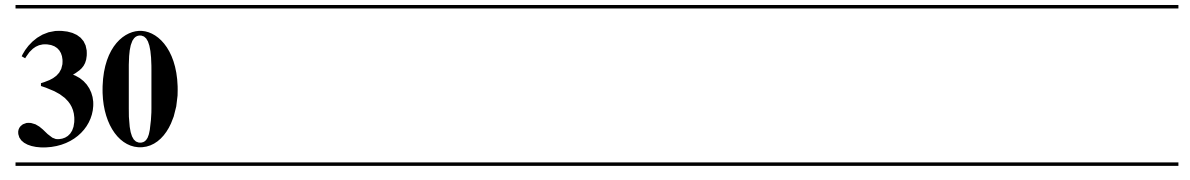

\title{
TESTING NATURAL BIOMATERIALS IN ANIMAL MODELS
}

\author{
Ana Costa-Pinto, ${ }^{1,2 *}$ Tírcia C. Santos, ${ }^{1,2 *}$ Nuno M. Neves, ${ }^{1,2}$ \\ AND RuI L. REIS ${ }^{1,2}$ \\ I3B's Research Group - University of Minho; Headquarters of the European Institute of \\ Excellence on Tissue Engineering and Regenerative Medicine, AvePark, Parque de \\ Ciência e Tecnologia, Zona Industrial da Gandra, 4805-017 Barco GMR - Portugal \\ ${ }^{2}$ ICVS/3B's - PT Government Associate Laboratory, Braga/Guimarães, Portugal
}

\subsection{LABORATORY ANIMALS AS TOOLS IN BIOMATERIALS TESTING}

Animal models have been extensively developed in the last decades in biomedical field. Their use has shown particular relevance in fields such as cell biology, genetics, anatomy and development, biochemistry, infection and immunity, cancer research, drugs and vaccine development, tissue engineering and regenerative medicine.

Despite major advances regarding in vitro models aiming to mimic the complexity and cellular interaction existing within tissues [1-3], in vivo testing is essential to safely investigate the biological performance of newly developed devices when implanted in a living system. A better characterization of such response at cellular and molecular level is required, and has been extensively investigated in the last decades [4-7]. However, the complexity of in vivo responses to implanted biomaterials renders this assessment a challenging issue to address.

\footnotetext{
* Both authors equally contributed for the writing of this book chapter.
}

Biomaterials from Nature for Advanced Devices and Therapies, First Edition. Edited by Nuno M. Neves and Rui L. Reis. (C) 2016 John Wiley \& Sons, Inc. Published 2016 by John Wiley \& Sons, Inc. 
TABLE 30.1 Criteria to consider when choosing the adequate animal model to characterize biomaterials

\begin{tabular}{lll}
\hline Biomaterial & Animal & Other \\
\hline Size & Species and strain & Housing conditions \\
Shape & Age & Technical expertise \\
Degradation time frame & Sex & Surgical procedure \\
Degradation products & Genetic background & Anesthetics \\
& Physiological status & Analgesics and antibiotics \\
& Metabolism & Clinical assessment \\
& Lesion to treat & Euthanasia/sacrifice methodologies \\
& Tissue to regenerate & Type of in vivo and post mortem analysis \\
& & Budget \\
\hline
\end{tabular}

As an experimental hypothesis is set, the animal model chosen should achieve the expected consistent answers, and also mimic the human clinical situation, which enables a correct extrapolation to be made.

The application of specific criteria to choose the adequate animal model is mandatory in biomedical research. Particularly in the field of testing biomaterials, it is of the utmost importance to have in consideration crucial aspects related to the biomaterial to be tested; these are summarized in Table 30.1. Additionally to these basic criteria, other important issues must be considered in functional animal models related to the specific tissue to regenerate, such as bone or cartilage, as will be discussed later in this chapter.

The basic criteria are all interconnected, and it is impossible to consider them separately when the researcher needs to choose the animal model to characterize a biomaterial. There are compendia that can guide researchers to select adequate models according to the specific aim of their research [8]. However, previous knowledge of animal science and welfare must be considered in order to accurately read and understand the information provided in these books.

The initial in vivo evaluation of biomaterials is usually, but not limited to, the assessment of the inflammatory and host response to the implanted material. At this stage of evaluation, the size and shape of the material are only important in considering a suitable animal to accommodate the implant, and in which a response can be observed and measured. On the other hand, when functional studies are considered, the size and particularly the shape must mimic the final aspect of the material to be used in human medicine as much as possible. For instance, this applies to biomaterials for tissue regeneration (further discussed in section 30.3: the cartilage and bone specific situations). In the evaluation of inflammation and host reaction induced by a biomaterial, it is also important to know the material degradation time frame, as well as its degradation products, that can induce toxicity in the host. Bearing in mind the specific characteristics of the produced biomaterial, one must then consider the different types of animals which may be adequate models for the study. Rodents are traditionally used to assess inflammation; however other species can be used. Age, sex, genetic background and metabolism must be considered when analysing the obtained 
results in order to have a better correspondence with humans. The pathophysiological status is also an important condition, mainly in functional evaluation of the biomaterial (further discussed in section 30.3); healthy vs ill (or induced pathology) animals, although for inflammation and host reaction evaluation, healthy animals must be used.

The lesion to treat and/or the tissue to regenerate are criteria that must be addressed when choosing the functional animal model (further discussed in section 30.3). Despite the issues related with the biomaterials to characterize and the animal itself, other considerations are important to deal with, such as housing conditions and technical expertise, surgical procedures, pharmacological conflicts (e.g. anaesthesia, analgesia and antibiotherapy may interfere in the host reaction evaluation), the method of killing at the endpoint of the experiment or eventual euthanasia procedure if needed at any time during the experiment. Also, the type of analysis required (in vivo and/or post mortem) may limit the choosing of the animal (e.g. for in vivo luminescence, large animals cannot be used and if histologic processing of the samples is required at the endpoint). Finally, the budget is a recurrent issue that may force researchers to choose less costly animals (e.g. rodents) in detriment of ovine or caprine species but, of course, the results must be interpreted accordingly.

\subsection{INFLAMMATION AND HOST REACTION}

The induced host tissue trauma and the inflammatory process resulting from the implantation of a medical device [9-12] are of utmost importance for a successful outcome. Features of a chronic inflammation are usually attributed to the host response towards the implant, while an early acute inflammatory response is mainly endorsed by the implantation procedure. Nevertheless, no matter what, the final purposes of inflammation are to destroy (or control) the invading agent, to initiate the repair process, and to re-establish tissue function as a continuous event $[11,13]$.

As a wound is created, coagulation takes place in the context of acute inflammation. Simultaneously, the complement system, which has the capability to distinguish 'self' from 'nonself' [14, 15] is activated [11, 16,17] (Fig. 30.1). The interaction of plasma proteins such as immunoglobulins [12] and fibrin $[11,12,16,17]$ with the surface of the material, or through an inadequate down-regulation of convertase, which enables $\mathrm{C} 3 \mathrm{~b}$ binding to plasma proteins, such as albumin, immunoglobulin $\mathrm{G}$ ( $\operatorname{IgG}$ ) and fibrinogen [17], is the main factor responsible for this activation (Fig. 30.1). In addition, adsorbed proteins onto the surface of the implanted materials act as strong chemoattractants to polymorphonuclear neutrophils at an early stage and blood monocytes within 24 hours (Fig. 30.1). Macrophages derived from blood monocytes continue the phagocytic work initiated by neutrophils $[11,18]$, although they might also act as antigen-presenting cells after processing the material [12], instigating specific immunological responses [11] in which lymphocytes also participate [11]. In general, the formation of foreign-body giant cells (FBGCs) indicates the transition to a chronic inflammatory process $[10,19]$. However, the same features may co-exist, attesting simultaneous development of acute and chronic inflammation [20]. As the FBGCs persist, unable to resolve the inflammation, cytokines and chemokines are 


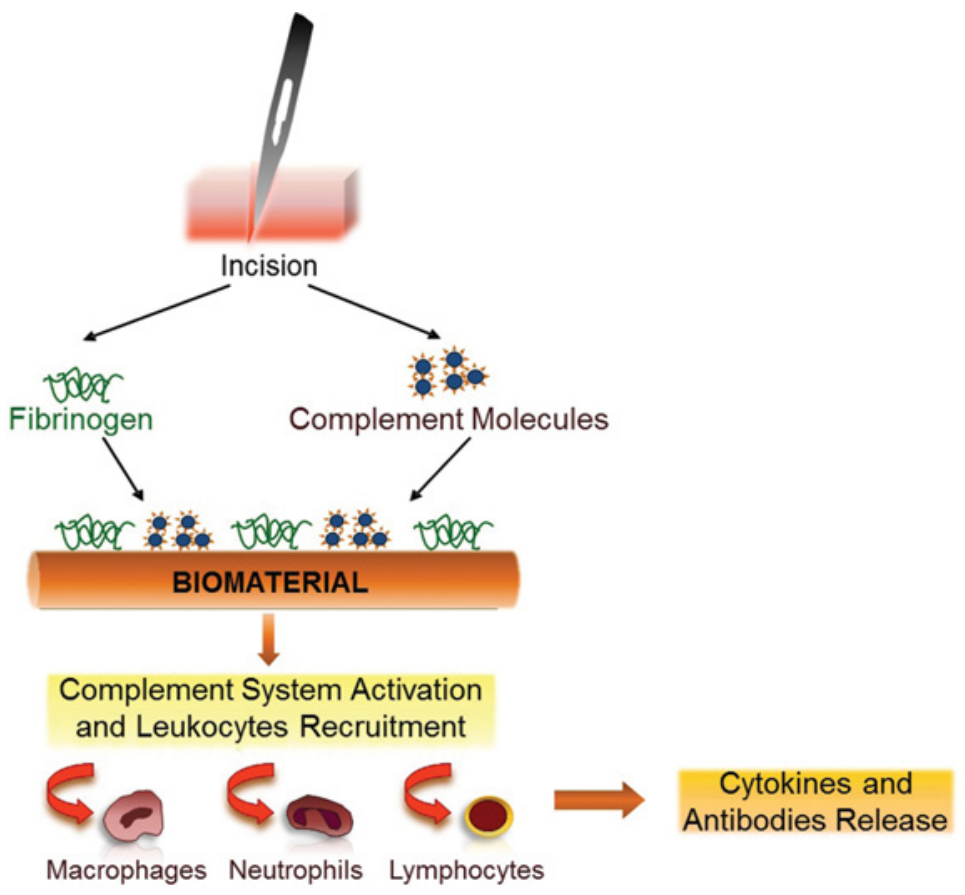

FIGURE 30.1 Schematic representation of the major events occurring from the creation of an incision, to host cell activation and cytokine release, which determine the ongoing inflammatory process. Once a wound is created, the microvasculature is affected and coagulation takes place in the context of acute inflammation. The complement system is activated mainly by the interaction of plasma proteins (e.g. immunoglobulins and fibrin) with the surface of the material, or through an inadequate down-regulation of convertase, which enables $\mathrm{C} 3 \mathrm{~b}$ binding to plasma proteins (e.g. albumin, immunoglobulin $\mathrm{G}(\mathrm{IgG})$ and fibrinogen). Then, the adsorbed proteins onto the surface of the implanted materials act as strong chemoattractants to polymorphonuclear neutrophils, blood monocytes which will attract lymphocytes and other inflammatory/immune cells. In their turn, these host cells secrete anti- and/or pro-inflammatory mediators (cytokines an antibodies) that will affect the ongoing inflammatory process.

released, inducing delayed-type hypersensitivity and forming a granuloma at the injury/implant site $[4,21,22]$. Granuloma formation is often a reason for implant rejection [23-27], or additionally may induce latent autoimmune diseases [28]. Some authors argue [23,29-31] that at the implant site, the foreign body induces chronic stress bringing forward the formation of granulomas. However, that is not always true and implants can be well-tolerated and integrated in the host tissue without eliciting a persistent acute inflammation [32]. Additionally, when collagen synthesis is likely to surpass its degradation [33], excessive fibrotic tissue surrounds the implant impeding the interaction of the host with the implanted material [22,33-35]. Therefore, thick fibrotic capsule formation is considered as a detrimental consequence of biomaterial implantation and a negative feature needed to be 
overcome, in order to improve the host reaction and eventually avoid rejection of the implant.

Ideally, an implanted biomaterial would interact with and integrate with the host tissue [36, 37], allowing the functional re-establishment and a complete recovery of the injured tissue. The resolution of inflammation with concomitant integration of the implant in the host tissue precedes the healing process. In a tissue engineered construct where the scaffolding material works as a temporary structure, the constant mutation of the implanted material will influence the reaction from the host. Additionally, the release of degradation products should not adversely interact with the host and should be physiologically discarded. Moreover, the presence of cells and bioactive agents influencing the properties of the polymeric structure complicate the ideal scenario and raises further concerns still to be overcome.

\subsubsection{Host Reaction Models}

The initial screening prior to the indication of biomaterials for clinical applications implies the validation of the safety of the biomaterials, as well as their degradation products when in contact with the host tissue. This evaluation has been traditionally performed in rodent (mice and rats) subcutaneous, intraperitoneal and intramuscular implantations models [38-44].

Subcutaneous, intramuscular and intraperitoneal murine models are useful tools to evaluate the effect of anti-inflammatory and inflammatory mediators, such as dexamethasone [45], nitric oxide [46], tumor necrosis factor-alpha (TNF- $\alpha$ ) and interferongamma (IFN- $\gamma$ ) [47], vascular endothelial growth factor (VEGF) and fibroblast growth factor (FGF) [48] towards host tissue at the implant site. Generally, assessed parameters are inflammatory tissue response, foreign body reaction (FBR), phagocytic potential of macrophages and eventual giant cell formation, fibrotic capsule formation and thickness, as well as vascularization in the surrounding tissue at the implantation site.

Despite the effort of researchers to use adequate models for their experiments, it is difficult to draw valuable information due to the differences in model and reaction mechanisms $[47,48]$, in carrier materials, or even in implanted material surface properties [45-48].

It is important to consider the different types of host reaction that can be elicited after the implantation of biomaterials, as was stated previously in this chapter. The primary acute inflammatory response is the most evaluated reaction after the implantation of biomaterials $[37,38,49,50]$. After this initial inflammatory reaction, different scenarios can occur:

(a) The implanted biomaterial degrades in a relatively short time frame (while the inflammatory response is still being observed) and the degradation products may cause inflammation themselves, or these products are metabolically excreted by the host;

(b) The biomaterial does not degrade in the course of the inflammatory reaction and is surrounded by a fibrotic capsule and no other interaction with host cells is observed (foreign body reaction) or; 
(c) The host does not surround the biomaterial with the fibrotic capsule, but is not able to degrade it, therefore two situations may arise:

(i) the host immune system is activated into setting up a chronic reaction or,

(ii) the acute inflammation persists and a nonhealing wound appears at the implantation site.

Most of the biomaterials that reach the in vivo evaluation stage are expected not to induce either chronic or persistent acute inflammatory responses. Regarding the degradation profile it may depend on the specific application. Nevertheless, the most frequently used host reaction models (subcutaneous, intramuscular and intraperitoneal) are also able to provide information on those types of reaction, particularly the chronic inflammatory response $[37,38,49,51]$ and the integration of the biomaterial within the host tissue after long periods of implantation [36,37,52-54]. Host reaction models must also be capable of providing information regarding local $[37,38,55]$ and systemic $[38,55,56]$ responses induced by the implantation of biomaterials. Regardless of the intended final application of biomaterials, subcutaneous and intramuscular implantation models offer information about the direct effect of the biomaterial at the implant site $[37,38,42,43,55]$, whereas intraperitoneal injections or implantations provide data on the effect in the abdominal organs, such as spleen, liver, kidney, mesenteric lymph nodes and related adipose tissue $[38,55,56]$, as an indication of the systemic influence of the biomaterials on the host. Moreover, intraperitoneal models are the most suitable for evaluating cell recruitment and activation status [56,57], at short $[56,58-60]$ and long periods $[54,56]$ of reaction. Besides injection of particle suspensions [55, 56], these models also permit materials implantation [54, 58-60], allowing establishment of a parallel between the implant/host interface analysis and the surrounding cellular milieu. The cytokine profile resulting from materials implantation is an example of an extremely powerful measure of this crosstalk. In fact, the cytokines in exudate samples are released by recruited cells which received the information from mediators, in its turn, secreted by surface adherent cells [61, 62]. Additionally, direct cell response to the implant surface may elicit the release of several reactive species, which either induce cell death or concomitant secretion of mediators, such as new reactive species.

Modification of the classical models of subcutaneous implantation, such as cage implants [7, 63], air pouches [41, 64, 65] or dorsal skinfold chambers [66] have also demonstrated reliable results regarding the interplay between direct and indirect material surface reactions. Cage implant models were shown to be useful for identifying recruited and adherent cell types [63] as well as macrophage fusion into FBGCs [7] and cytokine release [61] in response to implanted materials, either in rats $[7,63]$ or mice [61]. Moreover, the observation of leukocyte recruitment and accumulation was possible in a dorsal skin fold chamber [66], using intra-vital fluorescence and avoiding the killing of animals at different experimental time periods.

Additionally to the previously referred aspects, it has been mentioned that differences in host reaction can be related to sex dimorphism or with species, but few studies $[67,68]$ have really addressed these questions. Only subtle differences were observed between male and female, regarding the inflammatory cells pattern and connective tissue, as well as tumor necrosis factor alpha (TNF- $\alpha)$ and interleukin-1 beta 
(IL-1 $\beta$ ) secretion after subcutaneous implantation of a smooth peroxide-catalyzed polydimethylsiloxane (PMDS) in mice [67]. In terms of the species, Khouw and coworkers [68] showed that rats were able to overcome a foreign body reaction more effectively than mice, probably due to different recruitment kinetics, but stroma formation and calcification was more abundant in mice compared with rats [68].

Nevertheless, host reaction evaluation should not be limited to the assessment of inflammatory reaction, particularly if the developed device is aimed to remain in the host for longer periods and/or if it degrades during the implantation time. For these reasons, the immune response must also be evaluated before pursuing studies with the produced biomaterial. An adequate method to assess in vivo immune stimulation by biomaterials is to perform repetitive implants (rat subcutaneous [69] or intraperitonea 1 [54] model) and analyse the host-tissue response, immune cells and antibody production $[54,69]$. The development of surfaces with less immunogenic potential $[54,58,70-72]$ is one of the most important goals when producing biomaterials for biomedical applications. In fact, one of the researcher's great beliefs is that the implant/host interface is a key issue to identify tissue reaction to implants. Despite the effort in constructing and validating in vitro systems to predict the whole body reaction to the implants, there is not a reliable system that provides conclusive results on the assessment of the immunogenic potential of biomaterials for biomedical applications (see Chapter 26 for further details on immunogenic issues).

\subsection{ANIMAL MODELS FOR TISSUE ENGINEERING}

In vitro testing systems are inevitably limited in their capacity to recreate the complex in vivo environment. Therefore, these assays are unable to predict accurately the in vivo performance, particularly in the context of tissue engineering and regeneration of functional tissues. Factors such as the role of angiogenesis in newly formed tissue, immune reaction to implanted biomaterial and functional properties of the graft, are unable to be assessed in a an in vitro context. Thus, in a later stage of development it is critical to test the developed strategies in vivo.

The choice of an appropriate animal model will depend upon the question asked. Animal testing comprises a midway step between in vitro studies and human clinical trials, which precede real clinical application. Experimental design allows selection of species and standardized operative and analysis procedures. Animal models are simplified representations or analogues possessing the same or similar functions and structures as the system of study.

In vivo experimentation design in tissue engineering and regenerative medicine is demanding, being necessary to balance all variables and decide which animal model better suits the specific goals of the experiment. The maintenance of animals is expensive and variations within the same group may be larger compared with those that are found in small laboratory animals. A countless number of variables need to be addressed to assure that the chosen model is the most appropriate to evaluate a specific situation. Consequently, variables should be minimized and very well controlled to reduce random effects and to ensure as much as possible statistical significance. 
The variables are the physical condition of the animal (nutritional status, diet, age, sex), anesthetics and analgesics, type of defect (location, use of fixation) and, finally, the methodologies used to assess the sample collection and characterization.

Another important parameter is the inclusion of controls in the experimental design. These controls should contain a material already in clinical use, a control of an empty defect to prove that the bone defect is not able to regenerate by itself, and if it is using cells, the material without cells [73, 74].

The first approach generally chosen is the ectopic model in a small animal, usually rodents (mouse or rat). Ectopic is derived from the Greek word ektopos, meaning 'away from the place', and it refers to studies where ossification occurs outer the original places. On the other hand, orthotopic tissue formation is derived from the Greek word orthos, which means 'straight or right', and it refers to tissue formed at the normal place, i.e. bone in a bone location or cartilage in a cartilaginous area. The most simplistic of ectopic models is the subcutaneous implantation. Incisions are made in the dorsum of the animal, to prevent that animals disturbing their own sutures, in order to maintain the biomaterials/cells in place. The other type of ectopic model is the intramuscular implantation. Usually for small animals, use of the hind limb is recommended.

Moreover, if the aim is to evaluate tissue engineered constructs that include human cells, immunocompromised mice/rats are used, because these animals are genetically modified to not develop thymus, being unable to produce mature $\mathrm{T}$ lymphocytes, key immune cells in graft/implant rejection $[75,76]$.

Typically, researchers start with a smaller model like mouse or rat because of the low variations among animals, ease of handling and availability, cost efficiency and also because it is easier to compare results between a wealth of experiments reported in the literature. Independently of the model chosen, all results must be interpreted in light of the experimental model, and caution must be taken before extrapolating valid conclusions to humans.

In the next sub-chapters the animal models used for cartilage (section 30.3.1) and bone (section 30.3.2) regeneration will be described.

\subsubsection{Cartilage Tissue Engineering}

There are several animal models described in the literature used to conduct in vivo research on natural based biomaterials for cartilage regeneration. Cartilage can be classified, depending on the composition of the matrix, into elastic, fibro-cartilage, fibro-elastic and hyaline. A specialized type of hyaline cartilage, called 'articular cartilage', covers the joints. Hyaline cartilage provides a low friction surface, with high compressive strength and wear-resistant under normal circumstances [77].

The most important factors in the choice of an animal model for cartilage repair might be the size of the joint and cartilage thickness, as well as the animal size. The majority of the in vivo studies with rodents for cartilage tissue engineering, involves the use of heterotopic chondrogenesis models implanting biomaterials subcutaneously. Basically these models consist of making an incision in the dorsum of the animals and introducing the biomaterial (3D scaffold), or injecting it (gel) 
subcutaneously in each corner (up to four biomaterials are used) of mice. Immunocompromised animals enable conducting studies with allogeneic or xenogeneic cells with or without biomaterials. The small size of the joints and thickness of articular cartilage of these animals enable its use in other type of assay. Overall, rodents are cost-effective animal models that can be used for preliminary in vivo assessment of cartilage regeneration for further investigation in large animal models [78, 79]. In the natural biomaterials field, there are several reports of these types of animal model. A study with chitosan hydrogels injected subcutaneously in the dorsum of nude mice with or without newborn bovine primary articular chondrocytes, was allowed to develop for 7 or 9 weeks [80]. Wang et al. [79] used an alginate scaffold prepared using a microfluidic device seeded with porcine chondrocytes and implanted in the dorsal subcutaneous site of SCID mice. Controls were used by placing a blank scaffold without cell seeding in two animals per time point. The recipients were killed at 2, 4, and 6 weeks after transplantation [79]. In another study, recombinant human type I and II collagen gels were injected with or without bovine chondrocytes into the back of nude mice for 6 weeks [81].

Typically an induced cartilage defect is an osteochondral defect that consists of focal damaged areas of cartilage and injury of the adjacent subchondral bone. An example of this type of defect in rats, is the study of Dausse et al. [82], where the authors used full thickness cartilage defects of $1.3 \mathrm{~mm}$ drilled in femoral trochlea of rats. Filling of the resulting defect was achieved by injection of a hyaluronic acid hydrogel or insertion of alginate sponges, with or without autologous chondrocytes. The contralateral knee constituted an individual control of surgery, but without cartilage lesion [82].

In the early years of cartilage tissue engineering research, the rabbit was a popular model for osteochondral repair [83-85]. As the rodent model, these animals are genotypically similar, relatively inexpensive and easy to handle, which allows use of a significant number of animals in one experiment. Furthermore, rabbits reach early skeletal maturity at 9 months.

Cross-linked type I and type II collagen matrices, with and without attached chondroitin sulfate, were implanted into full-thickness defects in the femoral trochlea of adolescent rabbits. Two full-thickness defects of $4 \mathrm{~mm}$ per $1 \mathrm{~mm}$ were created in each trochlea. Empty defects were used as controls. The tissue response was evaluated at 4 and 12 weeks [85]. Autologous adipose stem cells and articular chondrocytes combined with gellan gum were injected in rabbit knee $4 \mathrm{~mm}$ per $1 \mathrm{~mm}$ full thickness size defects. Controls with defects filled with gellan gum without cells and empty defects were performed. The experiments were conducted for periods of up to 8 weeks with data collection points at 1,4 , and 8 weeks [83]. The lapine model is quite controversial, because of the endogenous healing potential of cartilage in rabbits [86]. Research on the cell origin of tissue repair in full-thickness cartilage defects concluded that the repair mechanism was mediated entirely by proliferation and differentiation of MSCs from bone marrow without participation from adjacent articular cartilage [87].

The pre-clinical evaluation of an articular regenerative strategy requires confirmation in large animals, such as sheep or goat, whose knee anatomy is similar to humans [88]. However, goats have cartilage proportions and subchondral bone involvement 
closer to humans [88]. The current surgical procedures to treat chondral lesions are aimed towards providing access for endogenous chondrogenic cells to the defect, such as microfracture. Subchondral bone is drilled and bone marrow cells derived from the bone marrow clot the defect, forming cartilage. However, the repair mechanism promotes the formation of nonfunctional fibrous tissue or fibrocartilage. Dorotka et al. [89] used an ovine model combined with microfracture treated with the matrix comprising type I, II, and III collagen, either unseeded or seeded with autologous chondrocytes. The animal study described here included 22 chondral defects in 11 sheep, divided into four treatment groups: $\mathrm{A}$ - microfracture and collagen matrix seeded with chondrocytes; $\mathrm{B}$ - microfracture and unseeded matrices; $\mathrm{C}$ - microfracture; D untreated defects. All animals were killed 16 weeks after implantation, and a histomorphometrical and qualitative evaluation of the defects was performed. Only the matrices seeded with autologous cells in combination with microfracture were able to facilitate the regeneration of hyaline-like cartilage. In a study by Lind and co-workers [90], a collagen membrane in combination with autologous chondrocytes or autologous cartilage chips were implanted for 4 months in a goat femoral condyle $6 \mathrm{~mm}$ full thickness cartilage defect model [90].

\subsubsection{Bone Tissue Engineering}

As previously described, in an ectopic study the constructs are implanted into a nonbone related anatomic location of the animal body, such as subcutaneously [91,92]. This model is also interesting for determining whether a scaffold has adequate properties namely porosity and interconnectivity, to allow tissue ingrowth and neovascularization. It is important to draw conclusions about the biodegradation of the implanted material, in terms of degradation products and also about the host immune response. The purpose of using such a model is to conclude about the ability of the constructs to form ectopic bone and also to verify osteoinductivity, i.e. the ability of the scaffold to induce proliferation of undifferentiated stem cells, as well as their differentiation into the osteogenic lineage $[75,76,78,93,94]$.

It is important to emphasize the concept of critical-sized defect (CSD), defined by an intraosseous wound that will not heal spontaneously during the lifetime of the animal [95]. However, the minimum size considered to be a critical size defect is not completely clear. The defect is not only defined by size, being dependent on diverse variables such as, type of species, anatomic location, among many others [96]. Guidelines are available for the dimensions of implants based on the size of the animal, type of bone chosen and implant design, in order to avoid pathological fracture of the test location [74]. Several types of bone defects can be used such as cranial, segmental, partially cortical and cancellous bone.

Bone regeneration must not only be evaluated in animals treated with the tissue engineering strategy, but also in sham-operated animals (negative controls) and in animals treated with autologous corticocancellous bone grafts (positive controls), which still remain the gold standard material in bone tissue engineering.

Different bone locations can be subjected to load or nonload bearing (e.g. femur or calvarial, respectively). The type of animal can be small (mouse or rat) [97-101] or 
large (rabbit, sheep, goat, dog or primate) [102-109]. One of the most frequently used models is the non-load bearing calvarial bone defect. This bone is flat, allowing the creation of a circular defect, by the use of a dental trephine with a dental drill against the superficial aspect of the calvarium. The dura mater and the overlying skin provide enough fixation of the scaffold and it is very important to keep the dura intact because the healing of a calvarial defect is dependent on the presence of this structure. The model has been systematically studied and is very well established [95, 110-112], using rat $[113,114]$ or mouse $[97,98]$. It can be also applied to larger animals, like rabbit [115-117] or sheep [118].

Natural biomaterials have been tested in rat calvarial bone defect models [114, $119,120]$. Chitosan and chitosan hydrochloride gels were tested in $8 \mathrm{~mm}$ calvaria defects in rats for 15 and 60 days. Blood clot was used as a control. The authors concluded that chitosan and chitosan hydrochloride biomaterials did not promote new bone formation in critical size defects made in rat's calvaria, with assorted degrees of inflammation [120]. The same animal model was used in another study with chitosan/tricalcium phosphate (TCP) sponges used as carriers for platelet derived growth factor (PDGF-BB) delivery. Chitosan/TCP sponges with and without PDGF-BB were implanted for 2 and 4 weeks, and the sponge carrier without PDGF-BB promoted osseous healing of the rat calvarial defects compared with controls (empty defects). The addition of PDGF-BB to the carrier further enhanced bone regeneration [119]. In another report, with the same animal model, fluorescent-labeled cells (MSCs) were encapsulated in a chitosan gel with or without bone morphogenic protein 2 (BMP-2). Five experimental groups were tested for 8 weeks: (1) chitosan gel; (2) gel loaded with MSCs; (3) gel loaded with BMP-2; (4) gel loaded with both MSC and BMP-2; (5) control group, the defect left untreated. The injectable form of chitosan gel, MSC, and BMP-2 enhanced more bone formation compared to the others [114].

Mouse cranial defect is also used to assess the capacity of natural biomaterials to promote bone regeneration $[75,76,98]$. Alginate scaffolds with or without octacalcium phosphate (OCP) were implanted in mouse calvaria critical-sized defects for 21 days [98]. Studies with cells, usually involve human derived cells, and for that reason immunocompromised mice (nude mice) are used. Undifferentiated human adipose tissue stem cells (hASCs) loaded in starch-polycaprolactone (SPCL) scaffolds were implanted in a critical-sized nude mice $4 \mathrm{~mm}$ calvarial defect, for 8 weeks. Cranial defects were either left empty, treated with an SPCL scaffold alone, or SPCL scaffold with hASCs. Improved new bone formation was observed in SPCL with hASCs compared with control groups that showed little healing [75]. In another report, CostaPinto et al. [76], used human bone marrow mesenchymal stem cells (hBMSCs) cultured in vitro for 2 weeks in osteogenic differentiation conditions chitosan fiber mesh scaffolds, and further implanted for 8 weeks in $5 \mathrm{~mm}$ cranial defects of nude mice. Scaffolds alone and empty defects were used as controls. Retrieved implants showed good integration with the surrounding tissue and significant bone formation, more evident for the scaffolds cultured and implanted with human cells (Fig. 30.2) [76].

However, since calvaria do not allow for the assessment of biomechanical loading at the interface of the implanted material, alternative anatomical load-bearing locations should be selected, such as mandible [121], femur [99, 100, 122] or tibia [123]. 

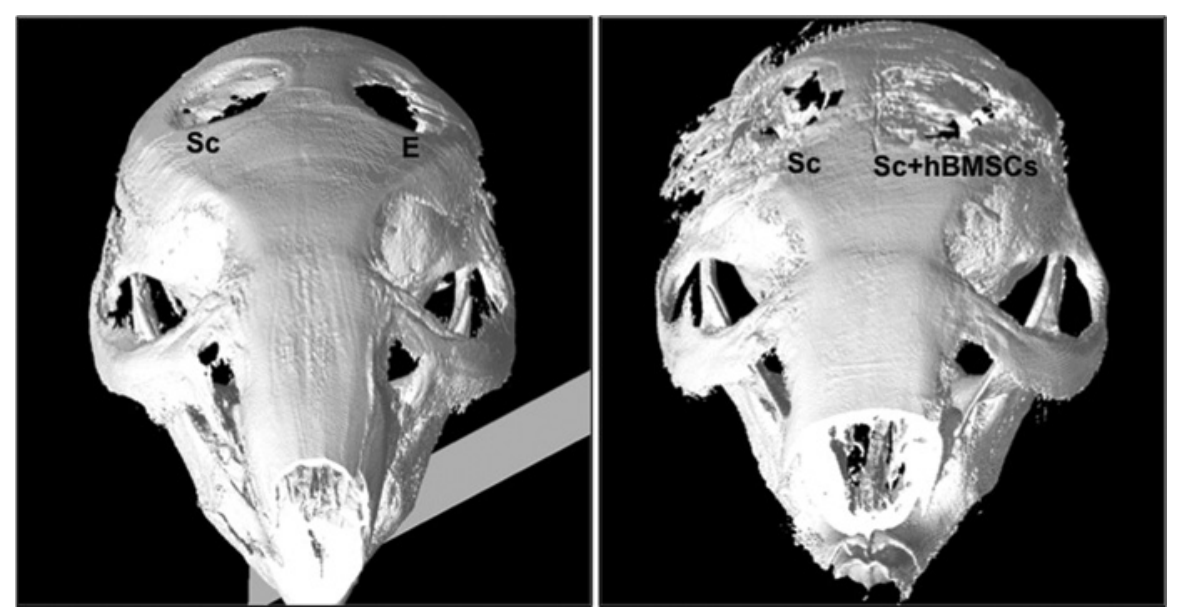

FIGURE 30.2 Micro-CT analysis of calvaria defects in nude mice after 8 weeks of implantation of chitosan-polybutylene succinate scaffolds with or without cells. E, empty; Sc, scaffold alone; Sc + MSCs, scaffolds with human bone marrow MSCs pre-cultured in vitro in osteogenic medium.

Natural biomaterial starch with polycaprolactone double layer scaffolds, functionalized with silanol groups (SPCL-Si) or without (SPCL), were tested in a mandibular defect rodent model [121]. Commercial collagen membranes and empty defects were used as positive and negative controls, respectively. After 8 weeks of implantation SPCL-Si scaffolds induced significantly more bone formation, when compared to collagen membrane and to the empty defects, although these had a similar performance when compared to the SPCL scaffolds [121]. In another study, starch-based scaffolds specifically, a blend of corn starch and ethylene-vinylalcohol (SEVA-C), the same composition coated with a biomimetic calcium phosphate $(\mathrm{Ca}-\mathrm{P})$ layer (SEVA$\mathrm{C} / \mathrm{CaP}$ ), and a blend of corn starch and cellulose acetate (SCA) were implanted into rat distal femurs proximal to the epiphyseal plate, for 1, 3, or 6 weeks. No bone contact was observed with SEVA-C at any time point, only transitory bone contact was observed with SEVA-C/CaP at 3 weeks, while SCA exhibited direct bone contact at 6 weeks [100]. Natural based silk fibroin scaffolds with hBMSCs pre-differentiated into the osteogenic lineage were evaluated in critical sized mid-femoral segmental defects in nude rats, for 8 weeks. Scaffolds without cells, and with undifferentiated cells, as well as empty defects were also tested. The group of animals with the silk scaffolds and pre-differentiated cells presented the best results in terms of bone formation [99].

The last stage of pre-clinical trials of a bone tissue engineering strategy should be performed in animals that are believed to be more similar to humans, in terms of metabolism, physiology, anatomy, etc. Small animals present several disadvantages in terms of anatomical disparity with humans, and difficulties in carrying out orthopedic procedures because of their small size and kinematic differences. For example, 
a femur defect in rats [124-126] is believed to heal faster that in larger animals [111]. However, in a study where the authors compared the bone ingrowth using the same chamber, in rats and in goats, no significant differences were observed between the two animals [127]. Surgeries involving load-bearing conditions, involving stabilization with internal or external fixation devices, require the presence of experts to perform the procedure. Larger animals are significantly more costly and more difficult to work with. Despite these issues, the final pre-clinical tests should be performed in large animals, subjected to load bearing comparable to the human case. For this purpose, sheep or goats may be good options. Both animals have a similar metabolism and bone remodeling rate to humans, as well as a comparable weight [106,118,128-130]. Large animals can be also used to test biomaterials in nonloading areas, such as intramuscularly or intra-cortically [128]. Biodegradable starch-based materials, starch/ethylene vinyl alcohol blend (SEVA-C) and a composite of SEVA$\mathrm{C}$ reinforced with hydroxyapatite (HA) particles were evaluated in intramuscular and intracortical (four holes of $5 \mathrm{~mm}$ diameter were drilled in the lateral cortex, where the scaffolds were pressed into the holes) bone implantation models in goats [128].

SPCL natural based scaffolds seeded and cultured with goat marrow stromal cells (GBMCs) 7 days in osteogenic differentiation conditions, were implanted in noncritical defects (diameter $6 \mathrm{~mm}$, depth $3 \mathrm{~mm}$ ) in the posterior femurs of goats, using an autologous model. Drill defects alone and defects filled with scaffolds without cells were used as controls [122].

\subsection{FINAL REMARKS}

From what has been previously pointed out it becomes relatively clear that the selection of an animal model it is not a straightforward issue. The particular case of inflammatory/host reaction evaluation adds additional challenges due to the high variance between animals in the same experiment, which is also a rather usual observation. A statistically representative approach, not only in the number of implanted materials, but also in the number of tested animals, is crucial to reduce the standard deviation for the results of the experiment, and to have confidence on the tissue response of that particular species to the implanted biomaterials. However, the increased discussion regarding the number of animals used in research, led to the establishment of models that avoid animal sacrifice and limited data outcome. Bioluminescence and transgenic animals represent a valuable combination in order to evaluate parameters, such as inflammation and vascularization. These models allow a long time analysis avoiding the killing of several animals at the intermediate time points. Within this effort to reduce the number of animals, Ho and co-workers [131] were able to assess the real-time in vivo inflammatory response to a subcutaneous implant of genipincross-linked gelatine, by in vivo bioluminescence, in a transgenic mouse model carrying the luciferase gene driven by $\mathrm{NF}-\kappa \mathrm{B}-$ responsive elements. The movement of host molecules is, in fact, an important issue to consider in the monitoring of the inflammatory/immune reaction to implanted biomaterials. In that particular case, the 
nuclear factor- $\kappa \mathrm{B}(\mathrm{NF}-\kappa \mathrm{B})$ is a nuclear transcription factor, critically involved in the regulation of inflammatory cytokine production and, consequently, in inflammation [132]. Likewise, in a dorsal skin fold chamber model performed in hamsters, is was also possible to determine in vivo vascularization with intravital fluorescence without killing the animal [66].

The shape and size of the biomaterial to be tested, as well as its final application, are important features to have in consideration when choosing the animal model. For example, for compact or scaffold material, subcutaneous or intramuscular implantation will be more suitable than the intraperitoneal implantation. This type of model would be more appropriate for testing the reaction of materials suspended in solutions, such as microparticles or nanoparticles.

The final intended use and function of the implanted biomaterial is also related to the degradability issue. Generally, natural polymers undergo enzymatic degradation and the degradation rate of a biomaterial is also linked to the type of response elicited by the host tissues. Phagocytic cells are normally able to remove debris from the tissue by engulfment and digestion, making the digestion of implanted materials an important issue to consider. In some cases it is not the biomaterial itself that induces a specific reaction, but the degradation products resulting from the concomitant action of the cells in the device.

Although challenging, more importantly in all animal use for biomedical research, in order to conduct the studies as accurately as possible, it is important to find or create a model, which allows the most precise answers to experimental questions, as well as a correct extrapolation to the human scenario.

No animal is ideal for every type of project in cartilage or bone research. As every animal model has its advantages and disadvantages, a comprehensive analysis of each available species needs to be conducted when planning an animal study. Cost effectiveness, anatomy, maturity, and joint biomechanics as well as postsurgical protocol must be taken into account. A model is relevant if experimental conditions and generated effects are linked. The experimental design must therefore include the innovating technique as well as negative and positive controls guaranteeing valid comparisons. The research question ultimately drives the choice of animal model.

\section{REFERENCES}

[1] Tseng, P. et al., Lab Chip 2014, 14, 1226.

[2] Vrana, N. E. et al., Tissue Eng Part B Rev 2013, 19, 529.

[3] Marx, U. et al., Altern Lab Anim 2012, 40, 235.

[4] Griffiths, M. M. et al., Methods 1996, 9, 295.

[5] Hunt, J. A. et al., Biomaterials 1997, 18, 1449.

[6] Hunt, J. A.; Williams, D. F. Biomaterials 1995, 16, 167.

[7] Kao, W. J.; Lee, D. Biomaterials 2001, 22, 2901.

[8] Rand, M. S. In Sourcebook of models for Biomedical Research; Conn, P. M., Ed.; Humana Press: New Jersey, 2008, p 9. 
[9] Mikos, A. G. et al., Adv Drug Deliv Rev 1998, 33, 111.

[10] Hunt, J. A. In Encyclopedia of Materials: Science and Technology; Elsevier Science Ltd.: 2001, p 4068.

[11] Stevens, A. et al., Wheater's Basic Histopathology: A Colour Atlas and Text; Fourth Edition ed.; Churchill Livingstone: Edinburgh, 2002.

[12] Williams, D. F. In Encyclopedia of Materials: Science and Technology; Elsevier Science Ltd.: 2001, p 542.

[13] Fantone, J.; Ward, P. In Pathology; Third edition ed.; Rubin, E., Farber, J., Eds.; Lippincott-Raven: 1999.

[14] Atkinson, J. P.; Farries, T. Immunol. Today 1987, 8, 212.

[15] Mollnes, T. E. Exp Clin Immunogenet 1997, 14, 24.

[16] Gorbet, M. B.; Sefton, M. V. Biomaterials 2004, 25, 5681.

[17] Nilsson, B. et al., Mol Immunol 2007, 44, 82.

[18] Bellingan, G. J. et al., J Immunol 1996, 157, 2577.

[19] Anderson, J. M. Curr Opin Hematol 2000, 7, 40.

[20] Lickorish, D. et al., Eur Cell Mater 2004, 8, 12.

[21] Goldsby, R. A. et al., Kuby Immunology; W. H. Freeman and Company: USA, 2000.

[22] Anderson, J. M. et al., Semin Immunol 2008, 20, 86.

[23] Kasper, C. S.; Chandler, P. J., Jr. Arch Dermatol 1994, 130, 48.

[24] Houpt, K. R.; Sontheimer, R. D. J Am Acad Dermatol 1994, 31, 626.

[25] Ossoff, R. H. et al., Ann Otol Rhinol Laryngol 1993, 102, 405.

[26] Mitnick, J. S. et al., Ann Plast Surg 1993, 31, 241.

[27] Hess, J. A. et al., Oral Surg Oral Med Oral Pathol 1982, 54, 499.

[28] Joosten, L. A. et al., Clin Exp Immunol 1994, 97, 204.

[29] Kaiser, W.; Zazgornik, J. Urologe A 1991, 30, 302.

[30] Overholt, M. A. et al., Cutis 1993, 51, 95.

[31] Wu, C. A. et al., J Biomed Mater Res A 2008.

[32] Anderson, J. M. Annual Review of Materials Research 2001, 31, 81.

[33] Wynn, T. A. J Pathol 2008, 214, 199.

[34] Martinez-Hernandez, A. In Pathology; Third edition ed.; Rubin, E., Farber, J., Eds.; Lippincott-Raven: 1999.

[35] Tang, L.; Eaton, J. W. Am J Clin Pathol 1995, 103, 466.

[36] Haisch, A. et al., Eur Arch Otorhinolaryngol 2005, 262, 338.

[37] Rhodes, N. P. et al., Biomacromolecules 2007, 8, 2733.

[38] Azab, A. K. et al., J Biomed Mater Res A 2007, 83, 414.

[39] Christenson, L. et al., J Biomed Mater Res 1991, 25, 1119.

[40] Kamath, S. et al., J Biomed Mater Res A 2008, 86, 617.

[41] Krause, T. J. et al., J Biomed Mater Res 1993, 27, 65.

[42] Meinel, L. et al., Biomaterials 2005, 26, 147.

[43] Mendez, J. A. et al., Biomaterials 2004, 25, 2381.

[44] Tang, L. et al., J Biomed Mater Res 1998, 41, 333.

[45] Hickey, T. et al., J Biomed Mater Res 2002, 61, 180. 
[46] Hetrick, E. M. et al., Biomaterials 2007, 28, 4571.

[47] Khouw, I. M. et al., J Biomed Mater Res 2001, 54, 234.

[48] Ravin, A. G. et al., J Biomed Mater Res 2001, 58, 313.

[49] Marques, A. P. et al., Macromolecular bioscience 2005, 5, 775.

[50] Spargo, B. J. et al., Biomaterials 1994, 15, 853.

[51] Kim, M. S. et al., Biomaterials 2007, 28, 5137.

[52] Ishii, D. et al., Biomacromolecules 2009, 10, 237.

[53] Matthews, B. D. et al., J Surg Res 2005, 123, 227.

[54] Schlosser, M. et al., J Biomed Mater Res 2002, 61, 450.

[55] De Souza, R. et al., Biomaterials 2009, 30, 3818.

[56] Tomazic-Jezic, V. J. et al., J Biomed Mater Res 2001, 55, 523.

[57] Bajaj, G. et al., PLoS One 2012, 7, e30899.

[58] Tang, L.; Eaton, J. W. J Exp Med 1993, 178, 2147.

[59] Lozano, F. S. et al., J Surg Res 2002, 107, 7.

[60] Busuttil, S. J. et al., J Thromb Haemost 2004, 2, 1798.

[61] Brodbeck, W. G. et al., J Biomed Mater Res A 2003, 64, 320.

[62] Robitaille, R. et al., Biomaterials 2005, 26, 4119.

[63] Brodbeck, W. G. et al., Proc Natl Acad Sci U S A 2002, 99, 10287.

[64] Hooper, K. A. et al., J Biomed Mater Res 2000, 50, 365.

[65] Wooley, P. H. et al., Biomaterials 2002, 23, 517.

[66] Laschke, M. W. et al., J Biomed Mater Res A 2005, 74, 696.

[67] Dalu, A. et al., Biomaterials 2000, 21, 1947.

[68] Khouw, I. M. et al., J Biomed Mater Res 2000, 52, 439.

[69] van Luyn, M. J. et al., Biomaterials 2001, 22, 1385.

[70] DeLustro, F. et al., Clin Orthop Relat Res 1990, 263.

[71] Hung, W. S. et al., J Biomed Mater Res 2001, 56, 93.

[72] Rodriguez, A. et al., J Biomed Mater Res A 2008, 85, 556.

[73] Kim, J.-H.; Kim, H.-W. Tissue Eng Regen Med 2013, 10, 310.

[74] Pearce, A. et al., Eur Cell Mater 2007, 2, 1.

[75] Carvalho, P. P. et al., Journal of Biomedical Materials Research Part A 2014, 102, 3102.

[76] Costa-Pinto, A. R. et al., Journal of Tissue Engineering and Regenerative Medicine 2012, 6, 21.

[77] Buckwalter, J. A.; Mankin, H. J. Instructional Course Lectures, The American Academy of Orthopaedic Surgeons - Articular Cartilage. Part I: Tissue Design and ChondrocyteMatrix Interactions ${ }^{* \dagger}$, 1997; Vol. 79.

[78] Cai, X. X. et al., Cell Biol. Int. 2007, 31, 776.

[79] Wang, C.-C. et al., Biomaterials 2012, 33, 120.

[80] Hoemann, C. D. et al., Osteoarthritis and Cartilage 2005, 13, 318.

[81] Pulkkinen, H. J. et al., Osteoarthritis and Cartilage 2010, 18, 1077.

[82] Dausse, Y. et al., Osteoarthritis and Cartilage 2003, 11, 16.

[83] Oliveira, J. et al., Journal of Orthopaedic Research 2010, 28, 1193. 
[84] Dashtdar, H. et al., Journal of Orthopaedic Research 2011, 29, 1336.

[85] Buma, P. et al., Biomaterials 2003, 24, 3255.

[86] Wei, X. et al., Journal of Biomedical Materials Research 1997, 34, 63.

[87] Shapiro, F. et al., Cell origin and differentiation in the repair of full-thickness defects of articular cartilage, 1993; Vol. 75.

[88] Ahern, B. J. et al., Osteoarthritis and Cartilage 2009, 17, 705.

[89] Dorotka, R. et al., Biomaterials 2005, 26, 3617.

[90] Lind, M.; Larsen, A. Connective Tissue Research 2008, 49, 437.

[91] Yu, B. et al., J. Nanomater. 2013, 8.

[92] Bhakta, G. et al., Acta Biomaterialia 2013, 9, 9098.

[93] Mendes, S. C. et al., J. Mater. Sci.-Mater. Med. 1998, 9, 855.

[94] Schantz, J. T. et al., Cell Transplant. 2002, 11, 125.

[95] Schmitz, J.; Hollinger, J. Clinical Orthopaedics and Related Research 1986, 205, 299.

[96] Lindsey, R. W. et al., Journal of Orthopaedic Research 2006, 24, 1438.

[97] Osathanon, T. et al., Biomaterials 2008, 29, 4091.

[98] Fuji, T. et al., Tissue Eng Part A 2009, 15, 3525.

[99] Meinel, L. et al., Bone 2006, 39, 922.

[100] Salgado, A. et al., J Biomed Mater Res A 2006, 80, 983.

[101] Zhang, Z.-Y. et al., Biomaterials 2010, 31, 608.

[102] Muzzarelli, R. A. et al., Biomaterials 1993, 14, 925.

[103] Kon, E. et al., Journal of Biomedical Materials Research 2000, 49, 328.

[104] Fialkov, J. A. et al., Journal of Craniofacial Surgery 2003, 14, 324.

[105] Nasser, N. J. et al., Injury 2005, 36, 1460.

[106] Mastrogiacomo, M. et al., Tissue Engineering 2006, 12, 1261.

[107] Zhu, L. et al., Tissue Engineering 2006, 12, 423.

[108] Takahashi, Y. et al., Tissue Engineering 2007, 13, 293.

[109] Umeda, H. et al., The Laryngoscope 2007, 117, 997.

[110] Hollinger, J. O.; Kleinschmidt, J. C. Journal of Craniofacial Surgery 1990, 1, 60.

[111] Yuehuei, H.; Freidman, R. Animal Models in Orthopaedic Research; 1st ed.; CRC press: Boca Raton, 1999.

[112] Montjovent, M. O. et al., Journal of Biomedical Materials Research Part A 2007, 83A, 41.

[113] Whang, K. et al., Tissue Engineering 1999, 5, 35.

[114] Stephan, S. J. et al., The Laryngoscope 2010, 120, 895.

[115] Kim, H. W. et al., J. Biomater. Appl. 2008, 22, 485.

[116] Kim, S. J. et al., Tissue Eng Regen Med 2009, 6, 63.

[117] Yeo, A. et al., Journal of Biomedical Materials Research Part A 2010, 93A, 1358.

[118] Giannoni, P. et al., J. Tissue Eng. Regen. Med. 2008, 2, 253.

[119] Lee, Y. M. et al., Journal of periodontology 2000, 71, 418.

[120] Spin-Neto, R. et al., J Biomed Mater Res A 2010, 93, 107.

[121] Requicha, J. F. et al., Journal of Orthopaedic Research 2014, 32, 904.

[122] Rodrigues, M. T. et al., J. Tissue Eng. Regen. Med. 2011, 5, 41. 
[123] Bumgardner, J. D. et al., Implant Dentistry 2007, 16, 66.

[124] Holmbom, J. et al., Journal of Biomedical Materials Research Part A 2005, 75A, 308.

[125] Yokoyama, A. et al., J. Biomed. Mater. Res. Part B 2005, 75B, 464.

[126] Chu, T. M. G. et al., Biomaterials 2007, 28, 459.

[127] van der Donk, S. et al., Comp Med. 2001, 51, 336.

[128] Mendes, S. et al., Biomaterials 2001, 22, 2057.

[129] Gugala, Z.; Gogolewski, S. Injury 2002, 33, 71.

[130] Kruyt, M. et al., J Orthop Res. 2004, 22, 544.

[131] Ho, T. Y. et al., Biomaterials 2007, 28, 4370.

[132] Bonizzi, G.; Karin, M. Trends Immunol 2004, 25, 280. 